\title{
AMR gradiometer for mine detection and sensing
}

\author{
Jan Vyhnánek ${ }^{\mathrm{a}}$, Michal Janošek ${ }^{\mathrm{a}}$, Pavel Ripka ${ }^{\mathrm{a}}$ \\ ${ }^{\mathrm{a}}$ Czech Technical University in Prague, Faculty of Electrical Engineering, Dept. of Measurement, Technická 2, \\ 16627 Prague, Czech Republic
}

\begin{abstract}
Anisotropic magnetoresistors (AMRs) are used to build an advanced mine detector. The sensing head involves a gradiometric pair of AMR sensors and a continuous-wave driven excitation coil, so the gradiometer is capable of detection ferromagnetic materials as well as diamagnetic metals. The sensors are specially arranged to suppress the large AC-excitation field, so the mine detector senses both $\mathrm{DC}$ and $\mathrm{AC}$ field gradient responses of the object of interest. Being limited by the sensor and electronics noise of $268 \mathrm{pT} / \sqrt{ } \mathrm{Hz}$ at $1 \mathrm{kHz}$, we were able to detect a $50 \times 50 \times 1.5 \mathrm{~mm}^{3}$ aluminum test object in a $20 \mathrm{~cm}$-depth using a $150 \mu \mathrm{T}, 1-\mathrm{kHz}$ excitation field.
\end{abstract}

Keywords: mine detection; magnetic sensors; AMR; gradiometer; eddy currents

\section{Introduction}

The mine detectors used for clearing landmines and other explosive remnants are nearly exclusively based on eddy currents and use induction coils for sensing the metallic parts of mines. They detect conducting objects very reliably, but they fail to discriminate dangerous objects from the scrap metal. The work of deminers is often very slow, as they have to carefully excavate each concealed metal object [1].

It would be desirable to use sensors with better spatial resolution and discriminate objects by the recognition of their shape and dimensions. The proposed gradiometer benefits from the simple design of commercially available AMR sensors and printed circuit board (PCB) technology. It can operate with a $1-\mathrm{kHz}$ continuous-wave excitation field, so nonmagnetic metals can be detected in the $\mathrm{AC}$ part of the gradiometer output in addition to ferromagnetic metals that affect the DC part. The gradiometric arrangement allows to measure the weak gradient field of the object of interest, while suppressing the strong excitation and Earth's field.

A similar mine detector with SDT sensors was presented in [2], however it worked with a pulsed field and the DC noise of the SDT sensors disqualifies them from the combined DC and AC response sensing. An AMR sensor working with a harmonic excitation field was used for non-destructive testing [3], but it did not solve the suppression of the Earth's magnetic field needed for the DC gradient sensing.

\section{Gradiometer principle}

AMRs of the KMZ51 type (NXP, ex. Philips) are arranged in the distance of $40 \mathrm{~mm}$, forming a vertical $\mathrm{dB}_{\mathrm{x}} / \mathrm{dx}$ gradiometer (Fig. 1). The two gradiometric sensors in a SO-8 package are soldered on the PCB together with signal preamplifiers (total gain of 1000x).

As a proper mine detector needs to sense diamagnetic metals too, we added a continuous-wave driven excitation coil and solved the most difficult part of suppressing the large excitation field, which is needed for the proper detection depth. The coil is symmetric to the gradiometric sensors and it is fed with a $1-\mathrm{kHz}$ harmonic signal resulting in an $\mathrm{AC}$ 
magnetic field of about $150 \mu \mathrm{T}$ amplitude. The first (measuring) AMR sensor is located on the sensitive side of the detector head and experiences the same excitation magnetic field as does the second (compensation) sensor. Without any deformation of the excitation or Earth's field the gradiometer response is near zero, limited by the gain of the PI controller in the feedback loop (Fig. 2).

Choosing the coil diameter is not a simple task: for a given excitation current, a small coil provides stronger magnetic fields to a small distance, while a larger coil delivers weaker fields to greater distances. The selected diameter of $27 \mathrm{~cm}$ should be suitable for both smaller and lager metal objects, this size is also common to commercial mine detectors.

\section{Gradiometer circuitry}

In order to assure the magnetic state of the AMR sensors and to improve the stability of its parameters, the AMR sensors are periodically remagnetized - "flipped" at $30 \mathrm{kHz}$. The sensor field response becomes modulated (Fig. 3), therefore the signal processing is shifted to a less noisy frequency band of the amplifiers. High flipping pulses (1.5 A peak) are used in order to lower the sensor noise [4] while keeping the maximum power dissipation by a low duty-cycle.

Synchronous demodulators with the reference signal of $30 \mathrm{kHz}$ provide reconstruction of the flipped output of the AMR sensors. The $30-\mathrm{kHz}$ demodulation stage is followed by a sampling circuit using a switched integrator, which is inactive in the noisy time intervals, where the sensor output is recovering after a flipping pulse [5]. The sensor connected to the feedback regulator is maintained in a zero magnetic field by the compensation coil current from DC up to $1 \mathrm{kHz}$. The compensating current flows through the serially-connected compensating coils of both AMR sensors, so the output of the second (measuring) sensor is proportional to the magnetic field gradient. Suppressing the excitation field and also the Earth's DC field by the compensator allows using higher excitation fields, therefore provides a reserve in DC gradient measurements and possibly an improvement in $\mathrm{S} / \mathrm{N}$ ratio.

There are four relevant outputs of the gradiometer: the DC field, DC field gradient and the AC field gradient decomposed into the real and imaginary parts. The DC field magnitude (homogeneous part compensating both sensors) is sensed on a shunt resistor in the feedback loop and can be used for correcting for the spurious sensitivity on the Earth's field due to nonorthogonalities of the gradiometer.

\section{System noise}

The noise of the measuring channel, which consists of a KMZ51 sensor, an AD621 preamplifier and a $30 \mathrm{kHz}$ demodulator, is shown in Fig. 4 - it was determined as $3.1 \mathrm{nT} / \sqrt{\mathrm{Hz}}$ @ $1 \mathrm{~Hz}$ and $268 \mathrm{pT} / \sqrt{\mathrm{Hz}} @ 1 \mathrm{kHz}$, respectively. When the feedback compensation is switched on (as in the normal operation), the noise increases by 19\%@1 Hz and 38\%@1 $\mathrm{kHz}$. This roughly corresponds to a noise increase due to adding an uncorrelated noise signal with the same standard deviation, which comes from the second (compensation) AMR sensor in our case. We could confirm the lack of correlation by the coherence measurement - the compensating current did not show any significant coherence with the signal from the measuring sensor (Fig. 5).

The 1/f noise of the sensor dominates in DC field measurements and decreases with the frequency. At higher frequencies, the system noise can be already limited by the instrumentation amplifier noise and resistive thermal noise of the bridge elements:

$$
B_{N}=\frac{1}{S} \sqrt{4 k T R+\mathrm{V}_{\mathrm{ni}}^{2}+\left(\mathrm{V}_{\mathrm{no}} / \mathrm{G}\right)^{2}+\left(\mathrm{I}_{\mathrm{ni}} \mathrm{R}\right)^{2} / 2} \text {. }
$$

The noise spectral density thus decreases with the lower resistance of the AMR bridge $(R)$ and higher sensitivity $(S)$, which can be augmented by a higher bridge bias voltage. The most 
critical parameters of the instrumentation amplifier are the input voltage noise $\left(V_{n i}\right)$, output voltage noise $\left(V_{n o}\right)$, current noise $\left(I_{n i}\right)$ and the gain $(G)$. For the KMZ51 and AD621 instrumentation amplifier, this would yield $137 \mathrm{pT} / \sqrt{ } \mathrm{Hz}$ noise spectral density which corresponds to the measured electronics noise - the $1-\mathrm{kHz}$ electronic noise was $141 \mathrm{pT} / \sqrt{\mathrm{Hz}}$ in this case, and was mainly limited by the $10 \mathrm{nV}$ noise of the instrumentation amplifier. As we did not notice any further improvement in the noise level of the KMZ51 when increasing its gain by a higher bridge supply voltage, we can conclude that even at $1 \mathrm{kHz}$ we were measuring the intrinsic magnetic noise of the sensor [6].

Further improvement in the noise performance is possible by using another type of AMR sensor [7]. With the AMR sensor HMC1001 and the low-noise instrumentation amplifier INA103 we were able to achieve $33 \mathrm{pT} / \sqrt{\mathrm{Hz}} @ 1 \mathrm{kHz}$, however the power consumption disqualifies these components from the application in an advanced handheld metal detector using multiple of these gradiometers in an array.

\section{Detection performance}

The gradiometer output was tested on aluminum and ferrous objects in different distances. The best approximation curve for both $\mathrm{AC}$ and $\mathrm{DC}$ gradient responses resulted in $\mathrm{x}^{-3}$. Compared to conventional metal detectors with an induction coil (e.g. Schiebel ATMID), the AMR gradiometer with KMZ51 sensors has still about a half of the detection depth. An aluminum object of $5 \times 5 \mathrm{~cm}$ could be recognized from the noise level at the gradiometer AC output up to the distance of $20 \mathrm{~cm}$. A ferrous nail $3 \mathrm{~cm}$ long and $3 \mathrm{~mm}$ thick could be recognized in the DC gradient signal up to the $10 \mathrm{~cm}$ distance.

Graphs of the spatial resolution (Fig. 6), measured with the $27 \mathrm{~cm}$-diameter excitation coil, confirm that the gradiometer responds to the local magnetic field disturbance, rather than the possible changes of the excitation field caused by the changing inductance of the coil. Therefore it is possible to use an array of gradiometers inside a single large coil (Fig. 7) - we built a prototype of such a metal detector which is a subject of further investigations [8].

However, as the AMR sensors in the gradiometer have their sensitive axes not perfectly coaxial, the gradiometer is still sensitive to homogeneous fields. Due to limited soldering accuracy and with no factory specifications of the sensitive axis deviation to the package frame of the sensor, the parasitic sensitivity will also differ for each gradiometer. For one gradiometer it was determined by calibrations to be $150 \mathrm{nT} / 50 \mu \mathrm{T}$ (output change caused by rotation in the Earth's field). The mine detector will be operated by sweeping in the horizontal plane; therefore the main part of the Earth's field is vertical: this linear dependence can be then used for correcting of the DC response using the homogeneous field magnitude (one of the four gradiometer outputs). The AC output of the gradiometer was found not to be affected by homogeneous fields, as the excitation field direction is stable.

\section{Conclusion}

The presented gradiometric mine detector uses AMR sensors of KMZ51 type for metal detection together with $1-\mathrm{kHz}$ continuous-wave excitation. As the gradient response of ferromagnetic and diamagnetic objects falls with distance approximately with a $1 / \mathrm{r}^{3}$ rule, the most limiting factor of the maximum detection depth is the sensor noise - in our case we can detect a $50 \times 50 \mathrm{~mm}$ aluminum plate up to $20 \mathrm{~cm}$ depth only being limited by the $268 \mathrm{pT} / \sqrt{\mathrm{Hz}}$ sensor and electronics noise at $1 \mathrm{kHz}$, which translates to $6.7 \mathrm{nT} / \mathrm{m} / \sqrt{\mathrm{Hz}}$ gradient noise. Further improvement of the detection depth is possible when using AMR sensors with a lower noise at the cost of increased current consumption. Prospectively, the high spatial resolution of AMR sensors should allow to recognize objects by using signals from a sensor array. 


\section{References:}

[1] D. Guelle, A. Smith, A. Lewis, T. Bloodworth, Metal detector handbook for humanitarian demining, European Communities, 2003.

[2] R.J. Wold, C.A. Nordman, E.M. Lavely, M. Tondra, E. Lange, M. Prouty, Development of a handheld mine detection system using a magnetoresistive sensor array, Proc. SPIE 1999; vol. 3710 (1), pp. 113-123.

[3] D.F. He, M. Tachiki, H. Itozaki, Highly sensitive anisotropic magnetoresistance magnetometer for Eddy-current nondestructive evaluation, Review of Scientific Instruments 2009, vol 80, pp. 036102-1 - 036102-2.

[4] H. Hauser, P.L. Fulmek, P. Haumer, M. Vopalensky, P. Ripka, Flipping field and stability in anisotropic magnetoresistive sensors, Sensors and Actuators 2003; vol. 106, pp. 121-125.

[5] P. Ripka, M. Vopalensky, A. Platil, M. Doscher, K.M.H. Lenssen, H. Hauser, AMR magnetometer, Journal of Magnetism and Magnetic Materials 2003; vol. 254-255, pp. 639641.

[6] N. Stutzke, S.E. Russek, D.P. Pappas, M. Tondra, Low-frequency noise measurements on commercial magnetoresistive sensors, Journal of Applied Physics 2005, vol 97, pp.

10Q107-1 - 10Q107-3.

[7] E. Zimmermann, A. Verweed, W. Glaas, A. Tillmann, A. Kemna, An AMR SensorBased Measurement System for Magnetoelectrical Resistivity Tomography, IEEE Sensors Journal 2005; vol. 5 (2), pp. 233-241.

[8] M. Janosek, J. Vyhnanek, P. Ripka, CW Metal Detector Based on AMR Sensor Array, proceedings of IEEE Sensors 2011 conference, unpublished. 
Jan Vyhnánek was born in Prague in 1987. Received the engineering degree from the Faculty of Electrical Engineering, Czech Technical University in Prague in 2011, currently a student of PhD at the Dept. of Measurement. His main fields of interest are magnetic sensors and their applications in detection of metal objects.

Michal Janošek, born in Varnsdorf in 1980, graduated from the Faculty of Electrical Engineering, CTU in Prague in 2007 at the Dept. of Measurement. He is working as a junior researcher and $\mathrm{PhD}$ candidate at the same department. His main research activity is the application of magnetic sensors in gradiometers and further development in PCB fluxgate sensors.

Pavel Ripka was born in Prague in 1959. He received an Engineering degree in 1984, a CSc (PhD equivalent) in 1989, Associate Prof. in 1996 and finally Prof. in 2002. He was elected the dean of the Faculty of Electrical Engineering, CTU in Prague in 2011. He continues to work at the Department of Measurement as a professor, lecturing in Measurements, Engineering Magnetism and Sensors. His main research interests are magnetic measurements and magnetic sensors, especially fluxgate. He is a co-author of 4 books and > 200 scientific papers. 
Fig. 1. - Gradiometer combined with the excitation coil form the detector head. The compensating and measuring sensors of the gradiometer are symmetrically placed on both sides of the excitation coil.

Fig. 2. - Functional diagram of the gradiometer operating in the excitation coil. Gradiometric function is obtained by connecting the compensation coils of the "measuring" and "compensated" sensors in series.

Fig. 3. - Sensor output (top trace) and the reference (middle) of the switched integrator suppressing the noisy intervals after flipping pulses (bottom trace).

Fig. 4. - Noise of the AMR sensor KMZ51 after the first demodulation (bottom trace), the influence of the feedback compensation (top trace). Noise level decrease above $1 \mathrm{kHz}$ is caused by the low pass filter of the demodulator.

Fig. 5. - Coherence of the compensating current and the measuring sensor output, the region of the near zero coherence continues up to $1 \mathrm{kHz}$.

Fig. 6. - Spatial resolution - a $10 \mathrm{~mm}$ diameter stainless steel sphere at distances of $4 \mathrm{~cm}, 6$ $\mathrm{cm}$ and $8 \mathrm{~cm}$ (AC gradiometer response).

Fig. 7. - The array of gradiometers with AMR sensors KMZ51 in a prototype mine detector 


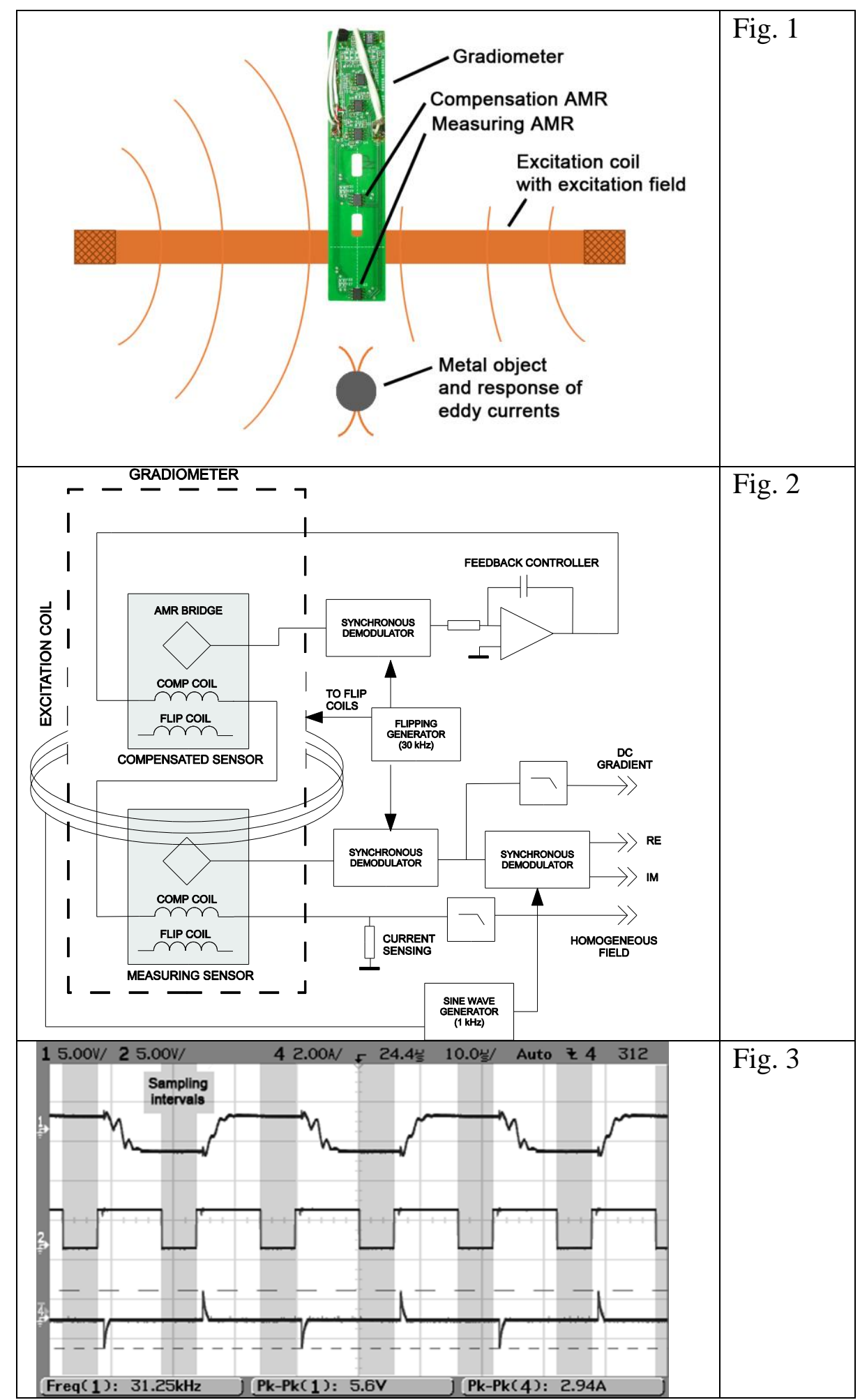




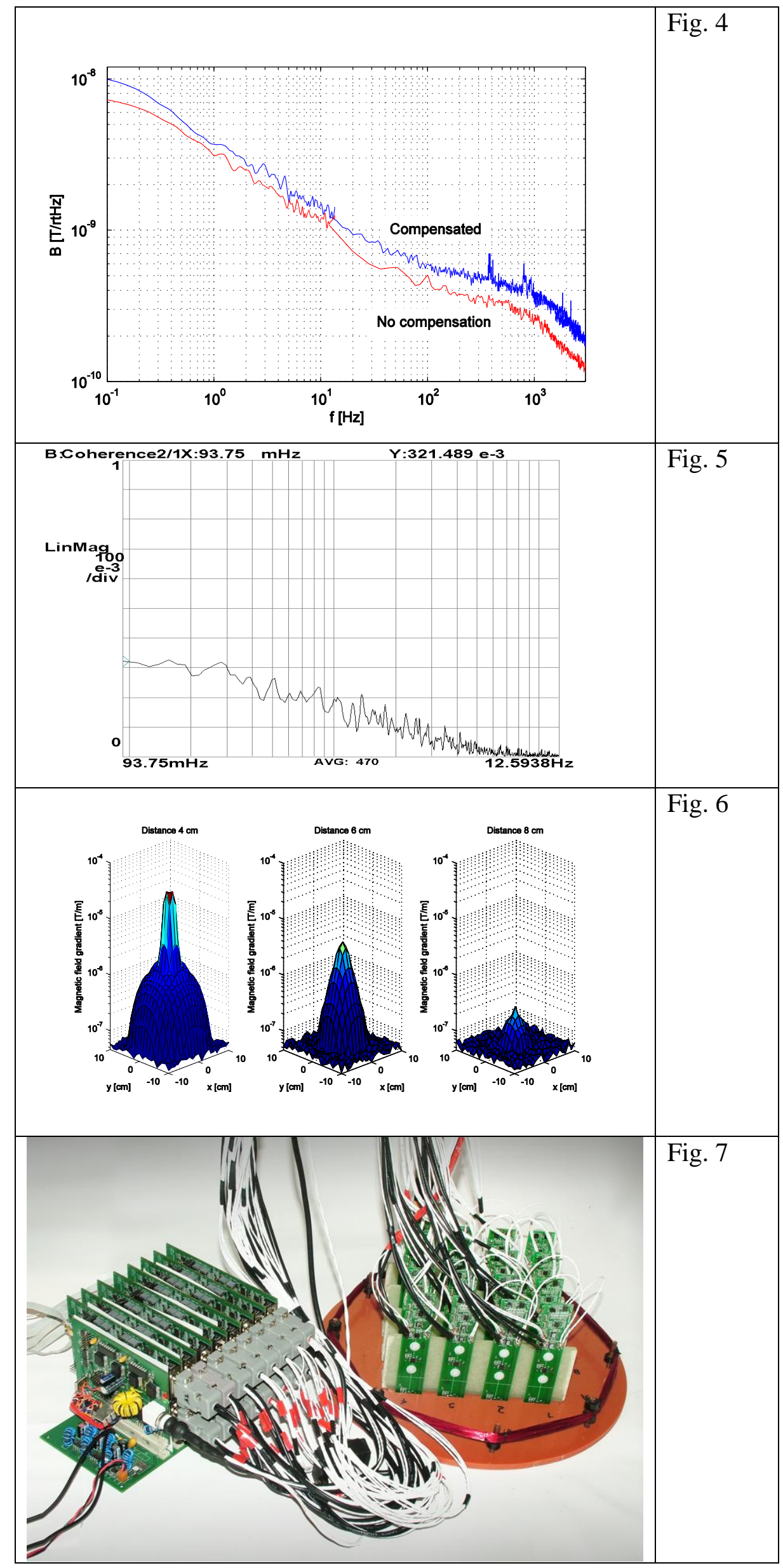

